\title{
Ask the experts: The challenges and benefits of flow chemistry to optimize drug development.
}

\section{Gernaey, Krist}

\section{Published in:}

Future Medicinal Chemistry

Link to article, DOI:

10.4155/FMC.12.106

Publication date:

2012

Document Version

Publisher's PDF, also known as Version of record

Link back to DTU Orbit

Citation (APA):

Gernaey, K. (2012). Ask the experts: The challenges and benefits of flow chemistry to optimize drug development. Future Medicinal Chemistry, 4(14), 1780-1781. https://doi.org/10.4155/FMC.12.106

\section{General rights}

Copyright and moral rights for the publications made accessible in the public portal are retained by the authors and/or other copyright owners and it is a condition of accessing publications that users recognise and abide by the legal requirements associated with these rights.

- Users may download and print one copy of any publication from the public portal for the purpose of private study or research.

- You may not further distribute the material or use it for any profit-making activity or commercial gain

- You may freely distribute the URL identifying the publication in the public portal 


\section{The challenges and benefits of flow chemistry to optimize drug development}

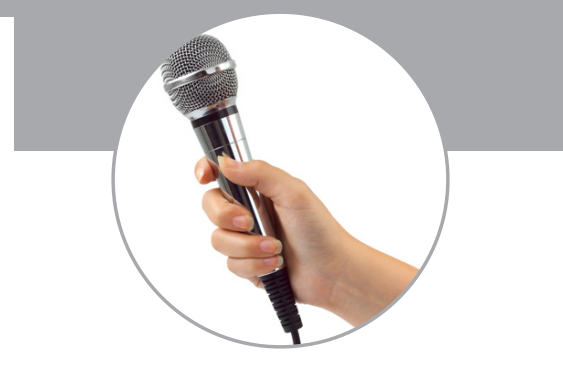

Against a backdrop of a struggling economic and regulatory climate, pharmaceutical companies have recently been forced to develop new ways to provide more efficient technology to meet the demands of a competitive drug industry. This issue, coupled with an increase in patent legislation and a rising generics market, makes these themes common issues in the growth of drug development. As a consequence, the importance of process chemistry and scale-up has never been more under the spotlight. Future Medicinal Chemistry wishes to share the thoughts and opinions of a variety of experts from this field, discussing issues concerning the use of flow chemistry to optimize drug development, the potential regulatory and environmental challenges faced with this, and whether the academic and industrial sectors could benefit from a more harmonized system relevant to process chemistry.

\section{- Neal Anderson; Anderson's Process Solutions LLC; nganderson@dishmail.net}

What attracted you to the field of process chemistry with regards to pharmaceuticals? To what extent has the importance of process chemistry changed in the past decade or so?

I was drawn to medicinal chemistry because I was fascinated with the chemistry of life, and I wanted to help people through developing APIs. In my studies, I found myself gravitating towards process chemistry, to thoroughly understand processes and optimize them for scale-up. My first job after

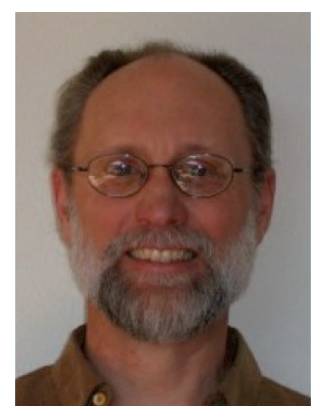
post-doc was in process chemistry, and I never looked back. Part of the attraction is that process chemists study compounds that are relatively likely to reach the market and help people.

In the 1980s process chemistry began to be recognized as a valuable piece of drug development, as more people realized that processes can rarely be scaled up tenfold to 1000 -fold without incident. Since that time more details of industrial process R\&D have progressively been divulged. For instance, in 1997 Organic Process Research \& Development began publication. In the past 2 years alone over 12 books have been published that will significantly impact industrial process R\&D. As the profit margins become narrower, people recognize that developing cost-effective processes is increasingly important.

The rising demand of generics and expiring IP have pushed pharmaceutical plants to become more creative and flexible in enhancing their productivity. How do you predict flow chemistry will aid this development? What are the merits, as well as flaws, of using this method of synthesis compared with traditional batch processing?

People develop continuous processes to make large quantities of materials for reasons of safety, quality, throughput and economics. Highly exothermic processes, such as nitrations, can be carried out safely by combining, at any instant, only small amounts of reactants. Some reactions, such as photolysis and sonication, can be efficiently carried out only under continuous flow conditions, while minimizing impurities and increasing quality and throughput.

Reactions requiring cryogenic temperatures in batch processing, such as Swern oxidations, may be run at moderate temperatures using very short reaction times; this can decrease the cost of manufacturing and allow manufacture to be carried out in facilities that do not have expensive Hastelloy reactors. An advantage of continuous flow operations is that once optimal conditions have been developed on a small scale further process development may not be needed to make larger quantities: the process can be run longer (scaled out) or conducted in parallel in multiple reactors (numbered up). Batch operations are appropriate for fine control of processes that require hours. Not every reaction is suitable for continuous processing.
FUTURE
SCIENCE 


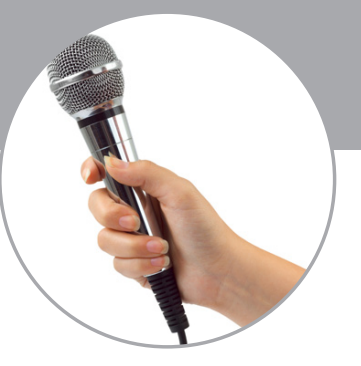

Q Some believe that the use of microreactors is integral to achieving high throughput in flow processing. However, are there any other methods that you believe to be equally as important?

Although microreactors are extremely effective in promoting efficient mixing and effective temperature control, the small volumes of microreactors and inherently high back pressures under rapid flow conditions can limit throughput. Microreactors have been built to incorporate many aspects of continuous flow chemistry that are familiar to chemical engineers. Reactors used to make larger amounts of material under continuous flow conditions include static mixers, continuous stirred tank reactors, and packed bed reactors containing insoluble catalysts such as $\mathrm{Pd} / \mathrm{C}$ and immobilized enzymes [1]. The pharmaceutical industry and related CMOs will develop more continuous processes using such reactors in the future.

Q Looking to the future, what are the key challenges facing flow and scale-up chemistry with respect to the pharmaceutical industry? Where do you predict the growth will lie within the next 5-10 years?

One challenge facing those wishing to develop continuous processes is how to define a batch for regulatory filings. A batch could be defined by the amount of starting materials, the output of product within a timeframe, or by the product from recrystallizing the total output of a process. Process validation requires understanding and demonstrating control of a process, and I believe continuous operations have been filed. Over the next 5-10 years the pharmaceutical industry will probably make greater use of continuous flow operations, not only for conducting reactions, but also for continuous separations and continuous crystallizations.

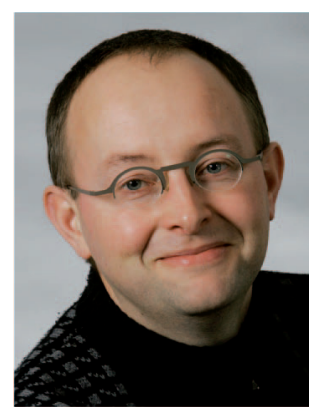

- Krist V Gernaey; Technical University of Denmark; kvg@kt.dtu.dk

Q To what extent has the importance of process chemistry changed in the past decade?

There is much more focus on process chemistry, compared with a decade ago. Different factors play a role there: the pharmaceutical industry has a focus on process optimization, driven by fierce competition, for example from generic producers; furthermore, there are problems to come up with new products. Process chemistry is one of the areas where production costs can be reduced, which is essential, especially when a product is no longer patent protected.

Q The rising demand of generics and expiring IP have pushed pharmaceutical plants to become more creative and flexible in enhancing their productivity recently. What are the merits of using this method of synthesis?

Flow chemistry/process intensification can potentially reduce the number of steps in a synthesis, thereby resulting in a greener process. In general, flow chemistry has the potential to reduce the process mass intensity considerably. Flow chemistry will, however, not solve all problems. Slow reactions fit better in a batch reactor. Solids are a problem as well, often excluding microreactors as a feasible flow synthesis solution.

Some state that the pharmaceutical industry will benefit from closer collaboration between the academic and industry sectors in order to facilitate drug discovery on an industrial scale. What are your opinions in regard to this?

I indeed think that a closer collaboration could lead to new views/novel approaches within drug discovery, thus leading to new products. One major obstacle is IPR, and especially how to share that IPR between industry and universities in a way that both parties are satisfied.

If this collaboration were to exist, what do you think would be the key areas that the two sectors should focus on to aid pharmaceutical production on an economic scale? Areas such as PAT methods and tools, and PAT implementation, development and use of computeraided systematic methods and tools to aid process design and the transfer of a process from the 
synthesis laboratory to the production plant (process systems engineering), the use of green solvents, and the use of biocatalysis.

2 Some believe that the use of microreactors is integral to achieve high throughput in flow processing. How can industrialists exploit the benefits of microreactors so that they are used to their full potential?

Microreactors have a huge potential, but are sensitive to, for example, solid formation during a reaction. Scaling out is not that evident either, assuming that a reasonably large production volume is to be produced. One should keep in mind that it is often easier, relatively, to work at meso-scale. In this way, one could in fact in many cases just move the meso-scale laboratory-scale reactor to the production plant, without having to worry about scaling up from laboratory to pilot to production scale.

Q How can factors such as biocatalysts, product recovery and purification enhance throughput? Biocatalysis could improve throughput because it can result in a $100 \%$ enantiomerically pure product. In this way, downstream operations can be simplified considerably, since no steps are needed to separate two enantiomers from each other.

\section{- Timothy F Jamison; Massachusetts Institute of Technology; tfj@mit.edu}

What attracted you to the field of process chemistry with regards to pharmaceuticals?

From my vantage point I find several aspects of process chemistry very interesting; in particular, the overarching aim of developing the most efficient synthesis by a combination of creativity and thorough investigations of the parameters and tolerances of each chemical and purification step.

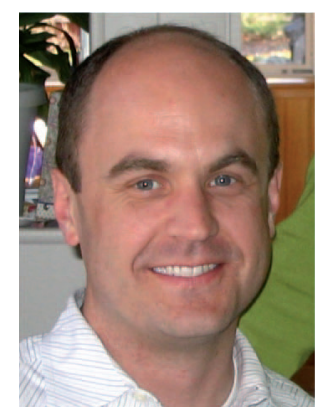

Q The rising demand of generics and expiring IP have pushed

pharmaceutical plants to become more creative and flexible in enhancing their productivity recently. What are the merits, as well as flaws, of using this method of synthesis compared with traditional batch processing?

Currently, there are several classes of transformations that derive significant and documented benefit by conducting them continuously, and there are several others where the benefits are less clear. Moreover, continuous methods enable certain types of chemical transformations that are not suited to batch scale-up and manufacturing (and thus not commonly used) may significantly enhance our armamentarium and have disruptive impacts.

Q In what ways does flow chemistry facilitate the ease of drug discovery with regards to enhanced reactivity and chemical space?

I think it remains to be seen, but it is logical to hypothesize that greater flexibility and new tools should have many positive impacts on both discovery and development.

\section{- Manfred Kircher; Chair of Advisory Board of Cluster Industrielle Biotechnologie (CLIB202I); kircher@clib202I.de}

Some state that the pharmaceutical industry will benefit from a closer collaboration between the academic and industry sectors in order to facilitate drug discovery on an industrial scale. What are your opinions on this proposal and has there been evidence so far? In such fields of cutting-edge science the pharmaceutical industry has cooperated with small- and middle-sized enterprises (SME) for many years. Successful business models either as fee for service or risk-sharing part-

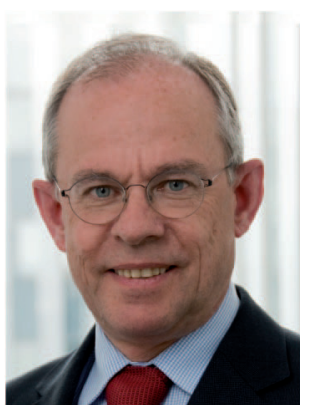
nering are established including upfront equity, milestone payments and

license fees. However, beside billion-dollar deals grabbing the headlines the relevance of academic research tends to be underestimated. It is not only the starting point of significant technologies for spin-offs and SME. Academic institutions are also sought-after development partners: in Germany 


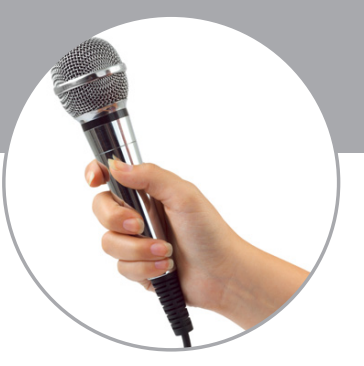

biotechnology companies close $56 \%$ of their partnering deals in research with academic institutions (development 47\%; validation 47\%; marketing 10\% [2012]).

What do you think are the key areas that the two sectors focus on to aid pharmaceutical production on an economic scale?

Academia is the partner of choice when it comes to the application of scientific findings - translating them into cost-efficient processes is the competence on the industrial side. For example, analyzing a biocatalyst and providing the tools to make it a production system is academia's strength but when it comes to optimize it according to the manufacturing cost structures industry performs better.

Q Do you think this partnership is possible? What issues arise with respect to this?

Academia is not only an attractive partner because of excellence in science and technology. A hidden value is its less distinctive orientation to specific industry sectors. Companies targeting energy, chemicals or pharmaceuticals address well-defined markets but may rely on similar or even identical technological platforms - sometimes without realizing it. For example, the underlying technologies to optimize bio-catalysts producing an energy carrier such as ethanol, a bulk chemical such as iso-butanol or a pharmaceutical active such as monoclonal antibodies are basically identical. Cooperation with academic research institutions helps to disseminate scientific progress across various industry sectors, thus pushing industrial application - assuming industries and academia are in regular contact.

It is obvious that sector-crossing partnering models are easier in regions where a critical number of multidisciplinary academic institutes meet multisector industries and SME along the value chain. Such a R\&D landscape is qualified for open innovation, cross-sectorial R\&D and academic/ industrial cooperation. If in addition stakeholders form a professional cluster capturing value out of academic technologies and shortening time to industrial markets, formation of unusual value chains is significantly promoted. The output of patents is a meaningful performance indicator for such cluster regions. Concerning life sciences IP, the global top ten are several US regions, with California's bay area leading, Tokyo, and in Europe the German state of Northrhine-Westfalia.

Looking to the future, what are the key challenges facing flow and scale-up chemistry with respect to the pharmaceutical industry?

The pharmaceutical industry faces three strategic challenges: an insufficient pipeline of new therapeutics in the fields of human medicine, drug delivery and tissue regeneration; a growing share of generics attended with increasing cost competition; and split-up of former blockbuster markets into fragmented patient groups due to personalized medicine.

Whereas the first topic asks for fundamentally new tools in R\&D to identify innovative NCE and modes of action, the other topics address pure cost issues in manufacturing the pharmaceutical active and the final drug as well as marketing it.

For sure, $R \& D$ targets must be carefully selected according to their indication's critical market size. Therefore, also diagnostics include the identification of minor gene variations gains relevance. Attention should be paid to R\&D platforms such as target screening, gene therapy and cell regeneration. As bio-pharmaceuticals take a growing share of the business (17\% in Germany; 2010) synthetic biology and cell-free biosynthesis will enable new efficient bio-catalysts for cost-cutting manufacturing processes.

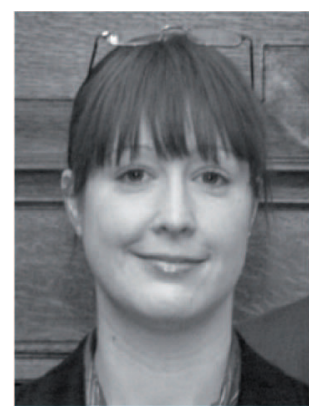

\section{- Charlotte Wiles; Chemtrix BV; c.wiles@chemtrix.com}

Q In what ways does flow chemistry facilitate the ease of drug discovery with regards to enhanced reactivity and chemical space? With speed being the driver of the discovery chemist, flow technology has the potential to aid the researcher by providing easy access to increased chemical work space. An example of this is the solvent freedom that can be accessed through the use of microreactors where these small volume, pressurized reactors allow the researcher to select the solvent properties to fit the chemistry rather than being limited to the temperature accessible at reflux. By enabling higher temperatures to be employed for low boiling, 
inexpensive solvents, there is potential to not only accelerate reactions, but to also reduce the time required to isolate and purify the target product.

In addition, the increased robustness associated with a flow process means that should the molecule prepared prove to be a viable candidate, a repeatable protocol already exists for the materials preparation at the $\mathrm{g}$ to $\mathrm{kg}$ scale - reducing the time taken to obtain material for further assessment and clinical trials.

In your opinion, how can the design of a flow chemical reactor be exploited to enhance cost-effectiveness, productivity and reduce waste?

When comparing flow chemistry with conventional batch research, it is clear to see that there are significant benefits to be harnessed from the use of flow reactors, particularly with regard to the rapid nature with which large volumes of data can be generated while utilizing only small quantities of reagents/catalysts and solvents. This is especially the case when employing microreactors where hundreds of reactions can be performed with only $\mathrm{mg} / \mathrm{ml}$ of material consumed in the full exploration of available chemical reaction space. From this information alone, the cost-effectiveness associated with a particular set of parameters can then be assessed ahead of deciding to take a project forward or confirming the need to perform further investigations into alternative parameters such as synthetic route, reagents, catalysts or technologies.

If the technology selected then forms parts of a scalable flow chemistry platform, the researcher has the ability to directly apply the conditions identified at the laboratory-scale for production of the given material with the confidence that there will not be the risk of a change in product quality or the failure to scale while maintaining productivity at the projected costs.

What steps do you believe have been taken recently to ensure the optimization and scale-up of flow chemistry processes? How does automation feature in this?

As with conventional batch processes, there are examples of flow reactions published where little process optimization is performed ahead of arriving at the 'optimal' conditions for material production; however, many researchers are embracing the opportunities that this technology brings for the generation of high quality, reliable/repeatable data and process understanding.

With this in mind, design of experiments is starting to feature in flow chemistry research and has the potential to reduce the time, number of experiments and material required to optimize a given process. To harness the full advantages of this approach, automation of flow reactor equipment is required and has recently been demonstrated by several flow chemistry equipment vendors. Ahead of scaling a synthetic flow process, it is imperative that the critical process parameters be identified in order to set the boundary conditions for a given process; with this information in hand, a process can be designed in such a way that monitoring of a set of system parameters allows monitoring of product quality.

Q Looking to the future, what are the key challenges facing flow and scale-up chemistry with respect to the pharmaceutical industry? Where do you predict growth will come within the next $5-10$ years?

While the advantages of flow chemistry are clear to any reader of synthetic journals, there is a learning curve associated with all new technologies and it is my opinion that students need to be taught the required skills to allow the pharmaceutical industry to benefit from flow. It is imperative that this discipline, along with other new technologies, be taught at an academic level utilizing in the first instance manual flow equipment, giving the next generation of chemists the skills required to truly benefit from automated flow platforms. In the next 5-10 years, I predict that growth in the pharmaceutical industry for the use of flow chemistry will be at the interface between drug discovery and process development; with the technique facilitating the preparation of the first milligrams of a material, then used to prepare grams to kilograms for clinical assessment.

For the technology to be harnessed more widely in production, the next decade needs to see an increase in the availability of validated production units that enable the scaling of processes from the early stages of milligram preparation to the production of materials in campaigns of tonne per annum. It also needs to be acknowledged that the reaction is not the only step to benefit from flow processing - work is required into the purification of continuously prepared active intermediates, 


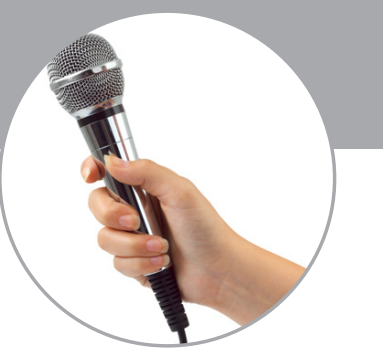

with cost savings to be made by combining improved synthetic methodology with less complex separation unit operations.

Presently, focus is being applied to the development of flow processes based on molecules that have already been identified through conventional discovery routes. Looking beyond the 5-10-year window, to see a molecule be taken from a flow based discovery platform through to continuous manufacturing will be the demonstrator that this technology has something special to offer the pharmaceutical industry.

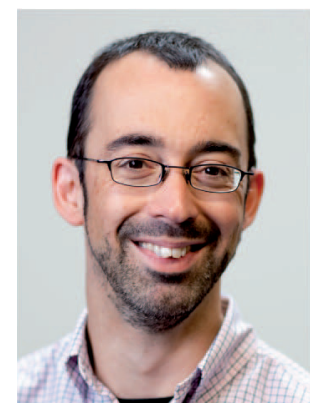

- Nicholas E Leadbeater; University of Connecticut; nicholas.leadbeater@uconn.edu

What attracted you to the field of process chemistry with regards to pharmaceuticals?

As a group from an academic setting, we are interested in interacting with process chemists and developing new methodologies for key synthetic transformations that they use.

Q The rising demand of generics and expiring IP have pushed pharmaceutical plants to become more creative and flexible in

enhancing their productivity recently. What are the merits, as well as flaws, of using this method of synthesis compared with traditional batch processing?

Flow chemistry offers a number of significant advantages over batch in many cases, although it is not the answer to everything. Reactions that require the use of hazardous reagents can benefit from flow processing since only a small amount of material is in the flow reactor at any one time. Also, the reagent can be generated in situ in the flow process, and excess can be quenched prior to leaving the flow reactor. Photochemical reactions can also benefit from flow processing - the high surface-area-to-volume ratio obtained when reagents are flowed through a tube next to the light source meaning that the light penetrates the entire reaction mixture effectively. Reactions involving reactive gases can be performed effectively in flow, this having both operational and (especially in the case of hydrogen) safety advantages. Some reactions can be performed effectively in flow by using a 'fast and hot' strategy, namely passing the reagents at a high flow rate through a heated zone at high temperature. The reaction can reach completion very rapidly and is out of the heated zone before significant byproduct formation occurs. At the other extreme, advantages have been seen flow chemistry performed at subambient temperatures. Organolithium reactions, for example, can be performed effectively and very rapidly.

Flow chemistry does have its disadvantages. If the reaction mixture is heterogeneous, it can often be difficult to process due to clogging of the reaction tubing. In addition, if the reaction is very slow, flow processing often does not bring any advantage over batch approaches.

2 Some state that the pharmaceutical industry will benefit from a closer collaboration between the academic and industry sectors in order to facilitate drug discovery on an industrial scale. What are your opinions in regard to this?

This is indeed the case. From our experience, working in close collaboration with industry has a mutually beneficial effect. The academic laboratory can bring new methodology to the industrial chemists. The industrial chemists can train the students in the academic laboratory in new techniques and allow them access to new technology. At the University of Connecticut we have taken advantage of this in developing a Masters program in which the student spends the first two years of the program working in the University and then spends 6 months working in a local pharmaceutical company, 3 months in medicinal chemistry and 3 months in process chemistry. This has reaped great rewards for the students involved in the program and also has allowed for new collaborations between the company and academics in the University.

Some believe that the use of microreactors is integral to achieve high throughput in flow processing. However, are there any disadvantages to this reaction system?

Microreactors have their advocates but also their detractors. They can easily block, which poses a real issue when working from a synthetic chemistry standpoint. However, they can (when properly 
designed) have outstanding heat transfer properties so can be used successfully for scale-up of highly exothermic reactions.

2 Microwave-promoted synthesis has become a growing area of interest for opening up new avenues of drug development, while providing a greener alternative to traditional flow processes. How will using this tool of synthesis advance the principles of green chemistry and engineering?

Microwave heating offers a fast and easy way to heat reaction mixtures. In essence, it can be used whenever a reaction requires heating. It also offers a safe way to operate at elevated temperatures and pressures and this can mean that reactions can often be performed very rapidly. From a synthetic chemistry standpoint, microwave heating is now widely used and it is finding applications in other areas of chemistry.

The greenness of the technology depends very much on the nature of the reaction. Just because a reaction is run in a microwave, it is not necessarily going to be any greener than when run using other methods. Also, microwave heating (at least on the small scale) is proven not to be energy efficient. As the scale is increased, it does become more energy efficient but energy consumption is definitely something that has to be taken into account.

Q In your opinion, how can the design of a flow chemical reactor be exploited to enhance cost-effectiveness, productivity and reduce waste?

Within both academic and industrial settings, chemists and chemical engineers are collaborating to come up with some innovative designs of flow reactors. This will definitely be something to watch closely in the coming months and years.

Q At what stage of the development process do you believe it best for regulatory bodies, such as the FDA, to approve new synthetic processes?

The FDA seems to be very pro continuous-processing so, as such, must assist chemists in filing new processes. Concepts such as 'batch size' when working in flow need to be resolved but this seems like a simple step.

\section{- Graham Sandford; University of Durham; graham.sandford@durham.ac.uk}

The rising demand of generics and expiring IP have pushed pharmaceutical plants to become more creative and flexible in enhancing their productivity recently. How do you predict flow chemistry will aid this development?

Continuous flow equipment can be used for a wide range of transformations rather than being limited to single chemical operations like a lot of dedicated batch plant equipment. The use of feedstocks and synthetic techniques that

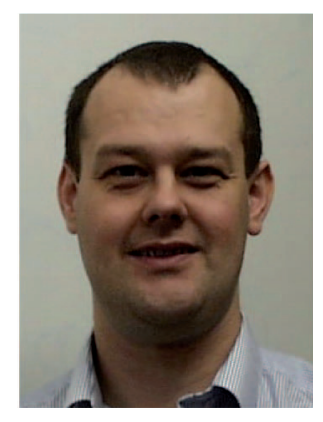
are not traditionally used in batch by pharmaceutical companies is also possible, extending the synthetic possibilities available to process chemists. This flexibility and concomitant low capital expenditure of flow equipment in comparison to dedicated pilot-stage facilities would be hugely beneficial in terms of resource savings.

In general, many flow processes described in the literature utilize traditional batch work-up procedures which negate many of the advantages of using continuous process techniques but this facet of flow chemistry is developing very rapidly to solve these problems.

Some state that the pharmaceutical industry will benefit from closer collaboration between the academic and industry sectors in order to facilitate drug discovery on an industrial scale. What are your opinions on this proposal and what, do you believe, would be the best steps to achieve this?

Of course, as an academic, I am probably a little biased in agreeing that pharmaceutical companies would benefit from more collaboration with academia but, in the UK, there has indeed been a long history of successful interactions between, for example, synthetic organic chemistry groups and drug discovery in pharmaceuticals. An effective academic/industry collaboration is one that 


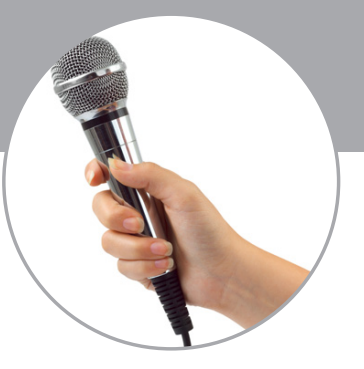

seeks to establish new principles or mechanistic insight by the academic partner with a wider drug discovery perspective provided by the industrialist. In my view, it is not the role of academia to invent new drugs with all the expense of testing, lead optimization but to develop new tools for pharmaceutical companies to exploit in facilitating drug discovery and manufacture. For example, at Durham University (UK), the fluorine group developed new direct fluorination methodology using fluorine gas as an academic exercise [2] and this was scaled up by industrial collaborators for use in the synthesis of a major pharmaceutical company (V-Fend, Pfizer) [3], exemplifying the mutual benefits of academia/pharmaceutical company collaboration.

Collaborations between academia/pharmaceutical companies are aided by joint government/pharmaceutical company funding and this should be developed further.

Q In your opinion, how can the design of a flow chemical reactor be exploited to enhance cost-effectiveness, productivity and to reduce waste?

There are many advantages associated with using flow reactors for chemical synthesis at both the laboratory and manufacturing stages and among those often discussed include high throughput, use of very small quantities of material when appropriate, reduced waste streams, low manufacturing, operation and maintenance costs, low power consumption, increased precision and accuracy and disposability. Miniaturization may also lead to increased performance of a system due to optimization of contact between reagents because of very rapid mixing in such devices. The concept of scale-up by running thousands of reactors in parallel is the most appealing, where bench operation would exactly mirror the manufacturing situation. There are additional special advantages in developing flow reactors for potentially hazardous processes, where the inventory of reactants is sufficiently small in any one reactor section, such that levels of control and safety could be achieved, which would not be possible by conventional means.

Q Looking to the future, what are the key challenges facing flow and scale-up chemistry with respect to the pharmaceutical industry?

Flow chemistry has, of course, been used for many years for the large scale production of commodity chemicals, but the adoption of flow techniques in the laboratory or small scale processes has been relatively slow. After all, batch processes have been used in the laboratory for several hundred years with extraordinary success so why change? Consequently, growth in flow chemistry stands the best chance of being utilized in processes that cannot effectively be carried out or are potentially dangerous in conventional batch techniques. This would allow pharmaceutical companies to manufacture new drugs using reagents that have not been traditionally used such as toxic reagents, very reactive reagents, relatively unstable reactive intermediates generated and used in situ in flow or processes not amenable to batch scale-up.

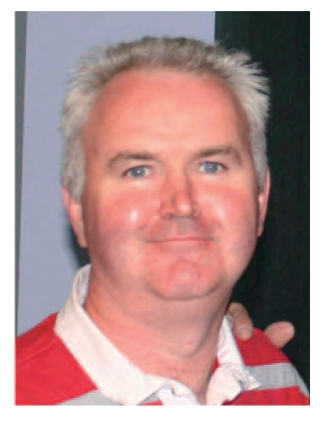

- Paul Richardson; Pfizer Inc.; paul.f.richardson@pfizer.com

Q What attracted you to the field of process chemistry with regards to pharmaceuticals?

I think I was initially attracted to pharmaceuticals in college pursuing my undergraduate degree in chemistry. We did an organic chemistry course on medicinal chemistry, and at that time, pharmaceuticals seemed the most glamorous of the potential career opportunities to go with after a chemistry degree. I think my focus leaned towards 'process chemistry' with my education following that. Although my $\mathrm{PhD}$ was a mix of methodology and synthesis, I then had two periods of postdoctoral study. The first was looking at using enzymes in organic synthesis, while the second looking at asymmetric catalysis at Scripps with Barry Sharpless. I think the latter really focused me on process chemistry. What was reinforced at that time was for a method to be widely embraced by the synthetic community, it had to be simple, well defined, and easily scaled. Initial work I did in that laboratory then looked at scaling up ligands for the asymmetric dihydroxylation reaction for commercial purposes. I worked in the UK for approximately 18 months, and then moved back to the USA to work for a start-up that based its chemistry initially on scalability of pharmacophoric building blocks. The key was finding robust processes to make these compounds on scale, and introduced 
and embraced the concept of click chemistry. I think this finalized my transition to process chemistry. The building block business progressed to a library business, and the company was bought by a genetics company, and transitioned to drug discovery. I have been in my current role in pharmaceuticals for 8 years.

The rising demand of generics and expiring IP have pushed pharmaceutical plants to become more creative and flexible in enhancing their productivity recently. How do you predict flow chemistry will aid this development? What are the merits, as well as flaws, of using this method of synthesis compared with traditional batch processing?

Let me preface this answer, and that of the subsequent ones regarding flow chemistry by saying that I am a proponent of flow chemistry. I believe that its strategic utilization can bring huge dividends to the pharmaceutical and chemical industries. I also believe that it is critical to recognize its scope and limitations, and one concern I have is that with its current high profile, people/scientists will believe that it's the solution for every problem. With this thought process, people will try to run reactions in flow, and with the first signs of failure, give up.

Second, I also think it is important to recognize that pharmaceutical syntheses are typically five to six steps, but it is not necessary to run the whole sequence in flow, but just those steps where flow chemistry brings a tangible benefit. I think several years ago when the topic rose to prominence, there was a perception that the footprint of production plants would be radically shrunk down, and all compounds would be made in flow. Clearly, this has not happened, and was never going to be the case, and there are only a couple of isolated examples were production processes have been re-tooled to partially run in a flow manner. Going forward with a greater emphasis on this tool, more production processes will go in place, but this will hopefully reflect an uptake in the technology in discovery chemistry and 'flow chemistry' steps developed at the early stage being taken through development. Although, the scaling of flow processes are probably not as simplistic (width obviously affects flow characteristics) as people are lead to believe they will probably be easier than scaling a batch process. Obvious advantages may well be reduced footprint of the plant (although one often omits the fact that purification/work-up is still required and space is needed here), increased production as in flow it's a measure of time (again a balance here between how much API is needed. If you develop dedicated flow equipment and you only need a small amount of API, is this worth it?), more precise reaction control, potential to heat to higher temperatures (potential to scale reactions previously run in the microwave) and greater safety (this to me is the greatest advantage).

Major challenges of flow chemistry is ability to handle heterogeneous reactions (getting things all in solution to prevent blockages), and also what reactions are applicable to flow from a kinetics standpoint.

Some state that the pharmaceutical industry will benefit from a closer collaboration between the academic and industry sectors in order to facilitate drug discovery on an industrial scale. What are your opinions on this proposal and what, do you believe, would be the best steps to achieve this?

I don't think there is anything particularly new to this proposal, and on paper it does appear to be a terrific idea. Often though, this is suggested as being an all encompassing panacea to solve bottleneck issues within drug discovery. There are, however, issues. Some common statements that are often heard is that 'innovation is typically done within academia', 'there is a gap between industry and academia', 'academia does not understand what industry does in drug discovery' or 'academia should do drug discovery alone as they would be more successful'. Obviously, there is always some basis of truth in all of these statements, and the fact of this usually depends on who is making the statement. My belief is that the process of drug discovery from an industry perspective would benefit from a 'true trusting partnership' with academic centers. However, the key words in that statement are 'true trusting partnership'. Often in the past the relationship has in general been perceived (and has often been the case) as the pharmaceutical industry funding academic research, which may only be of marginal interest to them. This is a wholly dissatisfying relationship in that it leads to the pharmaceutical partner rapidly losing interest and deprioritizing the collaboration, and the academic often takes the research in an entirely different direction. Afterwards this leaves a sour taste with both parties. At the current time, there is a move within pharmaceutical companies 
to locate close to academic hotbeds, which can only be good for collaboration. In addition, there is upfront communication on what both parties can bring to the table, and true efforts to set up collaborations on projects of mutual interest to both parties. Although my belief is that there should always be some separation between the interests of academia and industry, I believe that both can benefit in a collaborative sense from the skills, expertise and infrastructure of each to strongly influence drug discovery in the near future.

If closer collaboration were to exist, what do you think would be the key areas that the two sectors should focus on to aid pharmaceutical production on an economic scale?

One of the main areas I believe the pharmaceutical industry and academia should collaborate extensively on is target validation, although I believe this is beyond the scope of the question.

From a chemistry (and hence production) perspective, the three main areas in which I think collaboration should take place are:

- Scope of methodology - currently, academia is developing some absolutely super methodology such as $\mathrm{C}-\mathrm{H}$ activation chemistry that could potentially transform ongoing chemistry. However, the major critique from a pharmaceutical standpoint is the scope of the molecules this chemistry is performed on. Often, model compounds tend to lack heteroatoms, and not truly represent those being developed as potential bioactive substances.

- 'Green' chemistry methodology - again with methods developed, it is important to recognize what can and cannot be easily transferred for scale-up. Often green chemistry is touted as a key area, and this becomes more critical when one considers that by its nature 'green chemistry is good process chemistry'. Considerations such as toxicity of reagents, reduced waste and solvents should be thought about when developing methodology.

- Better communication of key problem areas. This has been better addressed in recent years, although often academic research chemists are surprised to learn that new effective scalable methods for 'amide bond' formation is still a need in industry.

Q In what ways does flow chemistry facilitate the ease of drug discovery with regards to enhanced reactivity and chemical space?

My own personal thought is that the major benefit of flow chemistry in terms of easing drug discovery is through enabling chemical space, which has previously been off limits due to safety concerns with reagents being utilized. Reactions using azides such as the Curtius reaction, ozonolysis or reactions with diazomethane are now potentially back within scope due to the ability to run these safely in flow. Flow also offers opportunities to utilize little used techniques. Photochemistry is very much in vogue due to only having to radiate a narrow flow cell. This enables unique transitions to take place (see, for example, its exploitation in Seeberger's very elegant Artemesinin work). Note here you haven't so much expanded chemical space as you can run this chemistry in classical batch mode. However, you have enabled the chemist to explore this by having a simpler avenue to do this. Electrochemistry is similar. For reactions carried out at high temperature, you effectively can superheat the solvents, although one can argue you are doing this in a microwave. However, the ability to scale-up flow is a critical advantage particularly if you consider microwave reactions to be simply enabled through heating.

What steps do you believe have been taken recently to ensure the optimization and scale-up of flow chemistry processes?

One area that I really like that has been developed is the introduction of relatively inexpensive, robust, easy to use equipment for laboratory use. This is somewhat akin to the introduction of microwave synthesis when initially this was introduced, it was considered to be a novelty, but now it is widely accepted. Walk-up kit takes the mystique out of flow chemistry. In fact, flow may even have a benefit here in that many discovery chemists are acquainted with the $\mathrm{H}$-cube for flow hydrogenation. This product served a niche to enable a potentially hazardous transformation to the laboratory chemist to be run safely. If chemists can realize this is essentially a flow process, then introducing the next generation of equipment should be more facile. 
Q Looking to the future, what are the key challenges facing flow and scale-up chemistry with respect to the pharmaceutical industry?

I think the key challenge for flow chemistry is to be widely implemented and demonstrate the tangible benefits that it is promoted to bring to the table. Improvements in flow chemistry to address its limitations would also be beneficial. I would very much like to see the transition of flow synthetic steps, from discovery all the way through the production continuum, become a realization. Again, I think it's important for the wider community to recognize the scope of this tool. Scale-up is always a challenge particularly with constant pressure to drive manufacturing costs down to stay competitive with generic production. Given the patent protection for a life cycle of a drug, one almost has to be constantly thinking about re-tooling the synthesis as soon as the compound reaches the market if one wants to be competitive once the patent life expires. Outsourcing is also a challenge for scale-up and flow chemistry, and one needs to be able to communicate effectively, and ensure both best practice and technologies are shared with one's partners.

\section{Financial \& competing interests disclosure}

The authors have no relevant affiliations or financial involvement with any organization or entity with a financial interest in or financial conflict with the subject matter or materials discussed in the manuscript. This includes employment, consultancies, honoraria, stock ownership or options, expert testimony, grants or patents received or pending, or royalties.

No writing assistance was utilized in the production of this manuscript.

\section{References}

1 Anderson NG. Using continuous processes to increase production. Org. Process Res. Dev. 16, 852-869 (2012).

2 Chambers RD, Greenhall MP, Hutchinson J. Direct fluorination of 1,3-dicarbonyl compounds. Tetrahedron 52, $1-8$ (1996).

3 Butters M, Ebbs J, Green SP et al. Process development of voriconazole: a novel broad-spectrum triazole antifungal agent. Org. Process Res. Dev. 5, 28-36 (2001). 